\title{
Cassiopeia A, Cygnus A, Taurus A, and Virgo A at ultra-low radio frequencies ${ }^{\star}$
}

\author{
F. de Gasperin ${ }^{1}$, J. Vink ${ }^{2,3,4}$, J. P. McKean ${ }^{5,6}$, A. Asgekar ${ }^{6,27}$, I. Avruch ${ }^{6}$, M. J. Bentum ${ }^{6,7}$, R. Blaauw ${ }^{6}$,
} A. Bonafede ${ }^{14,15,1}$, J. W. Broderick ${ }^{37}$, M. Brüggen ${ }^{1}$, F. Breitling ${ }^{24}$, W. N. Brouw ${ }^{5,6}$, H. R. Butcher ${ }^{26}$, B. Ciardi $^{28}$, V. Cuciti ${ }^{1}$, M. de $\operatorname{Vos}^{6}$, S. Duscha ${ }^{6}$, J. Eislöffel ${ }^{11}$, D. Engels ${ }^{1}$, R. A. Fallows ${ }^{6}$, T. M. O. Franzen ${ }^{6}$, M. A. Garrett ${ }^{18,8}$, A. W. Gunst ${ }^{6}$, J. Hörandel ${ }^{32,33,34}$, G. Heald ${ }^{25}$, M. Hoeft ${ }^{11}$, M. Iacobelli ${ }^{6}$, L. V. E. Koopmans ${ }^{5}$, A. Krankowski ${ }^{23}$, P. Maat ${ }^{6}$, G. Mann $^{24}$, M. Mevius ${ }^{6}$, G. Miley ${ }^{8}$, R. Morganti ${ }^{5,6}$, A. Nelles ${ }^{21,22}$, M. J. Norden ${ }^{6}$, A. R. Offringa ${ }^{6,5}$, E. Orrú ${ }^{6}$, H. Paas 5 , V. N. Pandey ${ }^{5,6}$, M. Pandey-Pommier ${ }^{30,31}$, R. Pekal ${ }^{36}$, R. Pizzo ${ }^{6}$, W. Reich ${ }^{35}$, A. Rowlinson ${ }^{2,6}$, H. J. A. Rottgering ${ }^{8}$, D. J. Schwarz ${ }^{29}$, A. Shulevski ${ }^{2}$, O. Smirnov ${ }^{9,10}$, C. Sobey ${ }^{25}$, M. Soida ${ }^{12}$, M. Steinmetz ${ }^{24}$, M. Tagger ${ }^{13}$, M. C. Toribio ${ }^{17}$, A. van Ardenne ${ }^{6}$, A. J. van der Horst ${ }^{19,20}$, M. P. van Haarlem ${ }^{6}$, R. J. van Weeren ${ }^{8}$, C. Vocks ${ }^{24}$, O. Wucknitz ${ }^{35}$, P. Zarka ${ }^{16}$, and P. Zucca ${ }^{6}$

(Affiliations can be found after the references)

Received 4 October 2019 / Accepted 18 February 2020

\begin{abstract}
Context. The four persistent radio sources in the northern sky with the highest flux density at metre wavelengths are Cassiopeia A, Cygnus A, Taurus A, and Virgo A; collectively they are called the A-team. Their flux densities at ultra-low frequencies $(<100 \mathrm{MHz})$ can reach several thousands of janskys, and they often contaminate observations of the low-frequency sky by interfering with image processing. Furthermore, these sources are foreground objects for all-sky observations hampering the study of faint signals, such as the cosmological $21 \mathrm{~cm}$ line from the epoch of reionisation.

Aims. We aim to produce robust models for the surface brightness emission as a function of frequency for the A-team sources at ultra-low frequencies. These models are needed for the calibration and imaging of wide-area surveys of the sky with low-frequency interferometers. This requires obtaining images at an angular resolution better than $15^{\prime \prime}$ with a high dynamic range and good image fidelity.

Methods. We observed the A-team with the Low Frequency Array (LOFAR) at frequencies between $30 \mathrm{MHz}$ and $77 \mathrm{MHz}$ using the Low Band Antenna system. We reduced the datasets and obtained an image for each A-team source.

Results. The paper presents the best models to date for the sources Cassiopeia A, Cygnus A, Taurus A, and Virgo A between $30 \mathrm{MHz}$ and $77 \mathrm{MHz}$. We were able to obtain the aimed resolution and dynamic range in all cases. Owing to its compactness and complexity, observations with the long baselines of the International LOFAR Telescope will be required to improve the source model for Cygnus A further.
\end{abstract}

Key words. radio continuum: general - techniques: interferometric

\section{Introduction}

Historically, the radio sources with the highest flux density in the sky were named after the constellation in which they were found followed by a letter starting with "A". They were then grouped in the so-called A-team ${ }^{1}$. In this work, we focus on the four persistent radio sources with the highest flux density (below $\mathrm{GHz}$ frequency) in the northern sky: Cassiopeia A, Cygnus A, Taurus A, and Virgo A (see Table 1), which are all very different in nature. Cassiopeia $\mathrm{A}$ is a prototypical supernova remnant, while a large fraction of the radio emission from Taurus A is powered by the central Crab pulsar and its associated shocked pulsar wind; Cygnus A is a very powerful Fanaroff-Riley (FR) type-II radio galaxy at the centre of a massive, merging galaxy cluster (Markevitch et al. 2002); and Virgo A is an amorphous radio source powered by a black hole with mass $M_{\mathrm{BH}}=(6.5 \pm$ $0.7) \times 10^{9} M_{\odot}($ Event Horizon Telescope Collaboration 2019) at the centre of a small, nearby galaxy cluster. Cygnus $A$ is at the distance of $232 \mathrm{Mpc}(z=0.056)$ and its radio power is

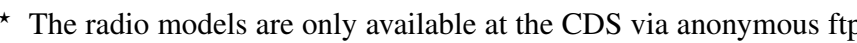
to cdsarc.u-strasbg. fr (130.79.128.5) or via http://cdsarc u-strasbg.fr/viz-bin/cat/J/A+A/635/A150

1 This is also a famous TV series from the 1980s.
}

$L_{1.4 \mathrm{GHz}} \simeq 1.2 \times 10^{28} \mathrm{~W} \mathrm{~Hz}^{-1}$ (assuming a flat $\Lambda \mathrm{CDM}$ cosmology with $H_{0}=71 \mathrm{~km} \mathrm{~s}^{-1} \mathrm{Mpc}^{-1}$ and $\left.\Omega_{\mathrm{M}}=0.27\right)$, which is among the highest registered for radio galaxies. Virgo $\mathrm{A}$ is at the centre of the closest galaxy cluster at the distance of $16.5 \mathrm{Mpc}(z=$ 0.00428 ) and its radio luminosity is $L_{1.4 \mathrm{GHz}} \simeq 8.3 \times 10^{24} \mathrm{~W} \mathrm{~Hz}^{-1}$. Cassiopeia A and Taurus A are Galactic sources at the distance of $3.4 \mathrm{kpc}$ (Reed et al. 1995) and $\sim 3 \mathrm{kpc}$ (Bailer-Jones et al. 2018), respectively.

These bright objects present a challenge for the calibration of radio interferometers, as their emission can leak into the primary beam side lobes and corrupt the dataset (e.g. Patil et al. 2017). This is especially relevant for low-frequency phased arrays, where the side lobes are less suppressed compared to dishbased instruments. A number of analysis techniques have been developed to account for the effect of the A-team in the data. A possibility is to predict the time-frequency regions of the observation where one side lobe of the beam crosses one of the A-team sources (Shimwell et al. 2017). If the predicted contaminating flux density is above a certain threshold, then that part of the data is discarded. This procedure is usually fast and it has been proven to be robust for observations with the High Band Antenna (HBA) system of the Low Frequency Array (LOFAR; van Haarlem et al. 2013), but it requires an accurate modelling 
of the primary beam side lobes. In the case of HBA observations, the amount of data loss is typically $5-10 \%$. Another technique that has been developed is the so-called demix (Van der Tol 2009). This technique requires high-frequency and time resolution data and it is conceptually similar to the "peeling" process (Noordam 2004). The dataset is phase-shifted towards the direction of the A-team source and is averaged down in time and frequency to smear all other sources. A calibration is then performed against a pre-existing model. Then, the model visibilities of the A-team source, corrupted with the solutions just obtained, is subtracted from the full-resolution dataset. When the A-team source is very close to a given target field $\left(<30^{\circ}\right)$, a standard peeling (Noordam 2004) or a multi-directional solve (e.g. Kazemi et al. 2011; Smirnov \& Tasse 2015) are viable solutions. In all the aforementioned cases, a good model for the surface brightness distribution of the A-team source is extremely valuable and, in many cases, essential.

Recently, the detection of a broad absorption profile, centred at $78 \pm 1 \mathrm{MHz}$ in the sky-averaged signal has been reported by Bowman et al. (2018). This boosted the interest in the ultra-low-frequency regime, driven by the possibility to detect neutral hydrogen during the cosmic dawn $(z \sim 30-15)$ and possibly even into the Dark Ages $(z \sim 200-30)$. The largest complication in these experiments is the subtraction of the strong astrophysical and instrumental foregrounds. The Galactic plane and the A-team sources are major contributors to the astrophysical foreground and a good model of these sources is paramount for their removal. Low-frequency, wide-field surveys have also renewed the interest of the broader scientific community (e.g. Shimwell et al. 2017; Intema et al. 2017; Hurley-Walker et al. 2017). For example, tracing cosmic rays (electrons) to the lowest energies provides insight into their inefficient acceleration mechanisms (e.g. de Gasperin et al. 2017). Low-frequency radio surveys can detect active galaxies in their late stages (e.g. Brienza et al. 2016), radio haloes and radio cluster shocks in merging clusters (e.g. Hoang et al. 2017), and also the highest redshift radio sources (e.g. Saxena et al. 2018). Again, our ability to carry out such surveys is limited by the extent that we can remove the contaminating emission from the bright A-team sources.

With the aim of determining accurate models for the surface brightness distribution of the A-team sources at low radio frequencies, we have carried out an imaging campaign with the Low Band Antenna (LBA) system of LOFAR, using the Dutch array. In Sect. 2, we describe the observations of the four sources and in Sect. 3 we discuss the data reduction. In Sect. 4, we describe the models that we are releasing to the astronomical community, and in Sect. 5 we briefly describe the main scientific outcome of this work.

\section{Observations}

The LOFAR (van Haarlem et al. 2013) radio interferometer is capable of observing at very low frequencies $(10-250 \mathrm{MHz})$. Each LOFAR station is composed of two sets of antennas: the LBA, which operates between 10 and $90 \mathrm{MHz}$, and the HBA, which operates between 110 and $250 \mathrm{MHz}$. Currently, LOFAR is composed of 24 core stations (CS; maximum baseline: $\sim 4 \mathrm{~km}$ ), 14 remote stations (RS; maximum baseline: $\sim 120 \mathrm{~km}$ ), and 14 international stations (IS; maximum baseline: $\sim 2000 \mathrm{~km}$, not used for this work).

For this paper, we took four separate LOFAR LBA observations, one for each A-team source. For these observations, we restricted our frequency range between $30 \mathrm{MHz}$ and $77 \mathrm{MHz}$. Below $30 \mathrm{MHz}$, RFI quickly dominates over the signal, while above $70 \mathrm{MHz}$ the LBA bandpass quickly drops. The datasets were divided into 244 sub-bands (SB) of $195.3 \mathrm{kHz}$ bandwidth each. The time resolution of all datasets was $1 \mathrm{~s}$ and the frequency resolution was 64 channels per $\mathrm{SB}(\sim 3 \mathrm{kHz})$. After radio frequency interference (RFI) excision (Offringa et al. 2010), the visibility datasets were averaged down to $10 \mathrm{~s}$ and 1 channel per SB. Some of the SBs were removed after inspection of the data if RFI was visible. We carried out the observations in LBA_OUTER mode, which uses only the outer half dipoles of each 96-antenna LBA field. This reduces the field of view to a full width at half maximum (FWHM) of $\sim 4^{\circ}$ at $60 \mathrm{MHz}$, and ignores the central dipoles where mutual coupling and unmodelled large-scale emission from the Galaxy make their calibration challenging. A summary of the observation parameters is given in Table 2 .

\section{Data reduction}

The data reduction follows roughly the strategy that has been outlined by de Gasperin et al. (2019), which was designed for point-like calibrator sources using the LBA system of LOFAR. All of our targets can also be considered bright calibrators, but the main difference is the complexity of their structure on $\sim 10^{\prime \prime}$ to arcminute scales. To compensate for this, we had to rely on a large number of self-calibration cycles to reconstruct the morphology of the sources.

\subsection{Initial model and flux scale}

The initial model for the self-calibration was taken from the literature or from archival data. Each model was rescaled to match the expected integrated flux density for a given frequency. The integrated flux density is modelled following Perley \& Butler (2017),

$\log (S[\mathrm{Jy}])=a_{0}+a_{1} \log (v[\mathrm{GHz}])+a_{2}[\log (v[\mathrm{GHz}])]^{2}+\ldots$,

where $v$ is the frequency and $A_{i}$ a set of coefficients. At these low frequencies Faraday depolarisation is very efficient, therefore all models are unpolarised. We now explain how we build up the initial model for each target.

Cassiopeia A. As a starting model, we used the LOFAR LBA image produced by Oonk et al. (2017). The model was rescaled to match the Perley \& Butler (2017) flux density using the parameters they derived as follows: $a_{0}=3.3584, a_{1}=$ $-0.7518, a_{2}=-0.0347$, and $a_{3}=-0.0705$. We note that the flux density of Cassiopeia A decreases with time (Baars et al. 1977; Vinyaikin 2014, and references therein).

Cygnus $A$. The initial model was taken from McKean et al. (2016) who observed this source using the LOFAR HBA system at frequencies between $109 \mathrm{MHz}$ and $183 \mathrm{MHz}$ that have an angular resolution of $3^{\prime \prime} .5$. The model has a higher resolution than what is needed to start our self-calibration process, and the source is known to undergo a rapid turnover in the bright hotspots below $100 \mathrm{MHz}$ (McKean et al. 2016). This makes the extrapolation of the HBA model just an approximation of the expected emission at LBA frequencies. The flux scale for Cygnus $\mathrm{A}$ has been estimated following Perley \& Butler (2017). The best fit is a polynomial function of the fifth order with parameters $a_{0}=3.3498, a_{1}=-1.0022, a_{2}=-0.2246$, $a_{3}=0.0227, a_{4}=0.0425$.

Taurus $A$. There was no prior model available for this object. However, this source has a compact bright component (the pulsar at the centre of the supernova remnant) that provides $\sim 10 \%$ 
Table 1. A-team: coordinates, flux densities, and sizes.

\begin{tabular}{|c|c|c|c|c|c|c|}
\hline \multirow[t]{2}{*}{ Source name } & \multicolumn{2}{|c|}{ Coordinates } & \multicolumn{3}{|c|}{ Flux density (Jy) } & \multirow{2}{*}{$\begin{array}{l}\operatorname{Size}^{(a)} \\
(\operatorname{arcmin})\end{array}$} \\
\hline & RA (J2000) & $\operatorname{Dec}(\mathrm{J} 2000)$ & at $50 \mathrm{MHz}$ & at $150 \mathrm{MHz}$ & at $1.4 \mathrm{GHz}$ & \\
\hline Cassiopeia A (3C 461) & $23^{\mathrm{h}} 23^{\mathrm{m}} 27 \mathrm{~s} .94$ & $+58^{\circ} 48^{\prime} 42^{\prime \prime} \cdot 4$ & 27104 & 9856 & 1768 & 7.4 \\
\hline Cygnus A (3C 405) & $19^{\mathrm{h}} 59^{\mathrm{m}} 28.35$ & $+40^{\circ} 44^{\prime} 02^{\prime \prime} .1$ & 22146 & 10713 & 1579 & 2.3 \\
\hline Taurus A (3C 144, M 1, Crab Nebula) & $05^{\mathrm{h}} 34^{\mathrm{m}} 31 \mathrm{~s} .97$ & $+22^{\circ} 00^{\prime} 52^{\prime \prime} .1$ & 2008 & 1368 & 829 & 7.9 \\
\hline Virgo A (3C 274, M 87) & $12^{\mathrm{h}} 30^{\mathrm{m}} 49 \mathrm{~s} .42$ & $+12^{\circ} 23^{\prime} 28^{\prime \prime} .0$ & 2635 & 1209 & 212 & 15.0 \\
\hline
\end{tabular}

Notes. ${ }^{(a)}$ Largest angular size as measured from LOFAR images at $50 \mathrm{MHz}$.

Table 2. Observations and image parameters.

\begin{tabular}{lcccccc}
\hline \hline Source & Obs. date & $\begin{array}{c}\text { Obs. length } \\
(\mathrm{h})\end{array}$ & Number of SBs & $\begin{array}{c}\text { Resolution } \\
(a) \\
(\operatorname{arcsec})\end{array}$ & $\begin{array}{c}\text { rms noise } \\
\text { (mJy beam }{ }^{-1} \text { ) }\end{array}$ & $\begin{array}{c}\text { Dynamic } \\
\text { range }\end{array}$ \\
\hline Cassiopeia A & 26-Aug.-2015 & 16 & 244 & $10^{\prime \prime} \times 7^{\prime \prime}$ & 11 & 7700 \\
Cygnus A & 04-May-2015 & 11 & 242 & $9^{\prime \prime} \times 6^{\prime \prime}$ & 40 & 18000 \\
Taurus A & 03-Mar.-2016 & 9 & 244 & $11^{\prime \prime} \times 8^{\prime \prime}$ & 6 & 35000 \\
Virgo A & 12-Apr.-2017 & 8 & 202 & $15^{\prime \prime} \times 12^{\prime \prime}$ & 5 & 18000 \\
\hline
\end{tabular}

Notes. ${ }^{(a)}$ At the mean frequency of $54 \mathrm{MHz}$.

of the total flux density, or about $300 \mathrm{Jy}$ at $50 \mathrm{MHz}$. We therefore started the self-calibration process assuming a point source model at the field centre and using only the shortest baselines (so that the entire source was seen as a point source) or the longest baselines (so that the extended component was resolved out and only the emission from the pulsar dominated the visibilities). In this way, we could obtain initial phase solutions for the LBA stations, which we then used to reconstruct the extended component of the source and continue the self-calibration process. The final model, with all of the components, was rescaled to match the Perley \& Butler (2017) scale using the parameters $a_{0}=2.9516$ and $a_{1}=-0.2173, a_{2}=-0.0473$, and $a_{3}=-0.0674$.

Virgo $A$. As a starting model, we used the low-resolution LOFAR LBA image presented by de Gasperin et al. (2012). The flux scale was set using a second order polynomial function with parameters $a_{0}=1226, a_{1}=-0.8116$, and $a_{2}=-0.0483$.

\subsection{Calibration}

The calibration procedure for all targets is described following the radio interferometer measurement equation (RIME) formalism (Hamaker et al. 1996; Smirnov 2011). First, all of the data points on baselines shorter than $30 \lambda$ were flagged to remove any extended structure associated with the Galactic plane. We also retained only the part of the observations where the targets were above $15^{\circ}$ elevation. Then, a first round of (direction independent) calibration was performed. Initially, for each SB we solved for a diagonal and a rotational matrix simultaneously, so that the Faraday rotation effect is channelled into the rotational matrix, while all other effects remain in the diagonal matrix. The latter was then used to compare the XX and YY solutions (the two diagonal elements of the matrix) and to extract from the phases the differential delay between the two polarisations. This effect was then applied together with the element beam model of the LOFAR LBA (van Haarlem et al. 2013). The data were then converted into a circular polarisation basis. In this basis, the effect of Faraday rotation can be described by a phase-only diagonal matrix with an opposite sign on the two circular polarisations. We solved per SB for a diagonal matrix and for each time step we fit the $\propto v^{-2}$ Faraday rotation effect on the difference between the two diagonal elements RR and LL. The dataset was then converted back to linear polarisation and corrected for Faraday rotation. Finally, a last diagonal matrix solve was performed at high frequency and time resolution to correct for ionospheric delay, clock errors, and the bandpass amplitude. These corrections were then applied and the dataset was ready for imaging and deconvolution.

\subsection{Imaging}

The imaging procedure for each self-calibration iteration was similar for all four targets. We used WSclean (Offringa et al. 2014) to perform the deconvolution. We weighted the visibility data using a Briggs (1995) weighting of -1 for Virgo A, -1.2 for Cassiopeia A and Taurus A, and -1.4 for Cygnus A. We chose these negative values to compensate for the large number of short baselines generated by the dense core of LOFAR. We used different weighting schemes to sample the different large and small scales of our targets. In all cases, we used multi-scale Clean with a large number of truncated Gaussian components, with scales up to the source extent. During imaging, the datasets were divided into 61 frequency blocks and imaged separately. All 61 images were combined to search for the peak emission to subtract during minor cycles. When the location of the clean component was determined, the brightness for that pixel was found for each image and a fourth order polynomial function was fitted through those measurements. These "smooth" components were then added to the model. The final images are shown in Fig. 1. The resolution of Cygnus A is higher than for the other sources to trace the more complex and compact structure for the source. However, the increased weighting of the data from the isolated LOFAR remote stations has an effect on the rms noise that, in this case, is four or more times higher than for the other sources. We did not perform any primary beam correction because, given the total extent of the sources, the average primary beam effect, even at the edges of our largest source, Virgo A, was always negligible $(<1 \%)$. In all cases, the target angular resolution for the models was achieved $\left(\theta_{\text {res }}<15^{\prime \prime}\right)$. 


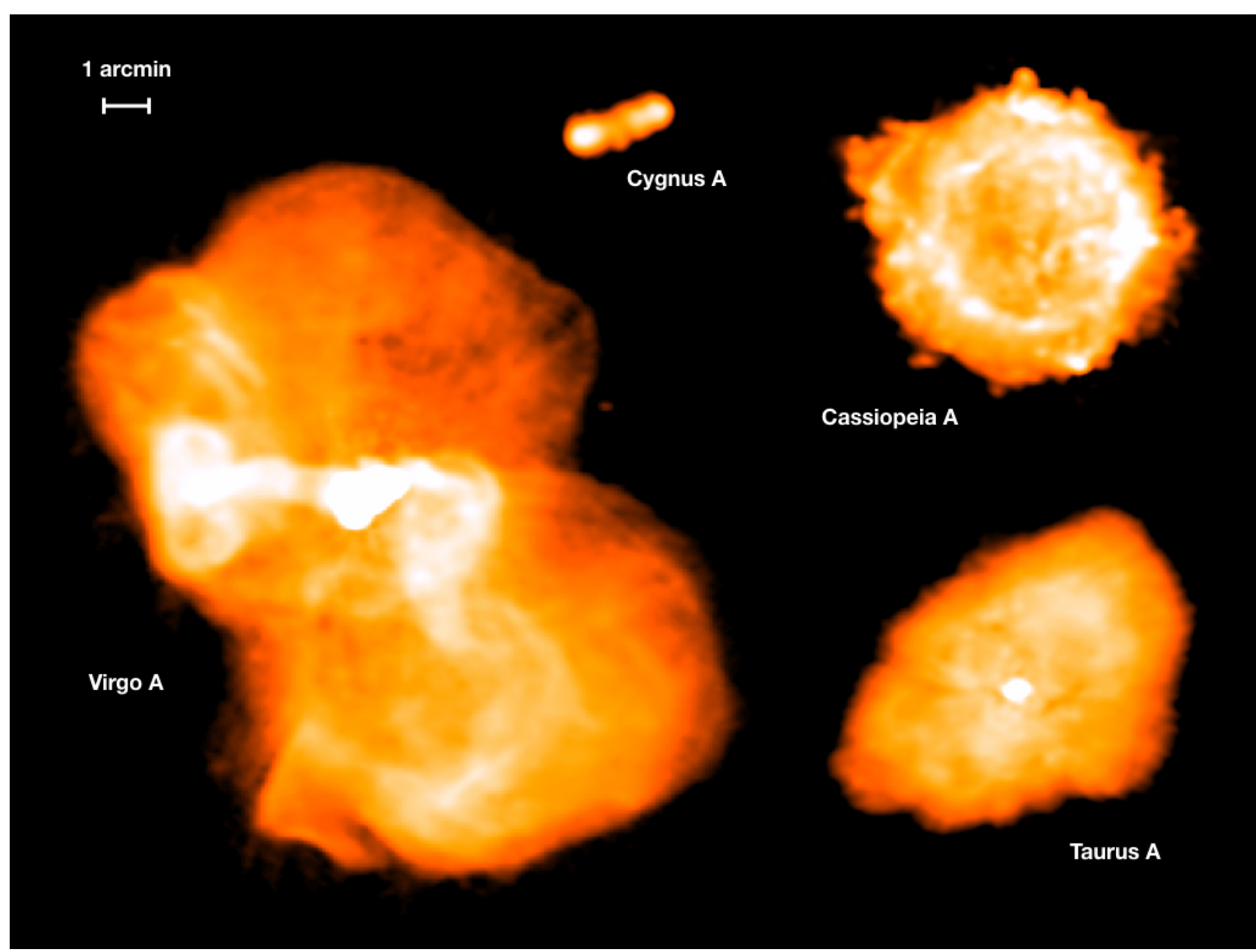

Fig. 1. Images of Cassiopeia A, Cygnus A, Taurus A, and Virgo A at a frequency of 50 MHz (using a bandwidth 30-77 MHz). Sources are scaled to show the correct apparent size ratio. The rms noise and resolution of each image are given in Table 2 .

\section{Models}

With this paper we provide the highest resolution models of the four A-team sources Cygnus A, Cassiopeia A, Taurus A, and Virgo A at ultra-low radio frequencies. The models are available at the CDS in two different formats that are compatible with WSclean (Offringa et al. 2014). The first is a set of model FITS files including the clean components at 61 different frequencies, equally divided in the frequency range from $30 \mathrm{MHz}$ to $77 \mathrm{MHz}$. The second is a text file including a list of clean components; the associated spectral shape is described by a seventh order polynomial function for Cygnus A, Cassiopeia A, and Taurus A, and by a fifth order polynomial function for Virgo A (see Table 3). Each clean component is one line of the file ${ }^{2}$. Some aspects to note: the type of clean component can only be "POINT" (for pointlike components) and "GAUSSIAN" for extended components. In the second case, the MajorAxis and MinorAxis are saved to represent the FWHM of the component. The I column represents the flux density in Jy at the reference frequency. The SpectralIndex column shows the coefficients of the polynomial function when normalised to the reference frequency. The polynomial

2 The data format is explained in detail at https://sourceforge. net/p/wsclean/wiki/ComponentList/ function is given by

$S_{v}=I+C_{0}\left(v / v_{0}-1\right)+C_{1}\left(v / v_{0}-1\right)^{2}+\ldots$,

where $I$ is the Stokes total intensity value, $v_{0}$ is the reference frequency, and $C_{0}, C_{1}, \ldots$ are the coefficients saved in the SpectralIndex column. The -1 within round brackets is necessary to let the assumed Stokes $I$ be the correct value at the reference frequency. Currently, all Gaussian clean components are circular, that is, the MajorAxis and MinorAxis are the same. We also provide a low-resolution model in text-file format obtained by re-imaging the data at $45^{\prime \prime}$ resolution. These models have fewer clean components and can be efficiently used in arrays with more compact configurations.

With these models the A-team sources can also be used as calibrators for ultra-low-frequency observations. However, if the sources are strongly resolved, then the flux density on the longest baselines might not be enough. In these cases, fainter but more compact sources such as 3c 196, 3c 380, or 2c 295 are preferred.

\section{Discussion and conclusions}

We obtained data for the four radio sources with highest flux density in the northern sky using the LOFAR LBA system 
Table 3. Two example lines from the clean component list files.

\begin{tabular}{lcccccccc}
\hline \hline Name & Type & RA & Dec & $\begin{array}{c}I \\
(\mathrm{Jy})\end{array}$ & SpectralIndex & $\begin{array}{c}\text { Ref.Frequency } \\
(\mathrm{Hz})\end{array}$ & $\begin{array}{c}\text { MajorAxis } \\
(\operatorname{arcsec})\end{array}$ & $\begin{array}{c}\text { MinorAxis } \\
(\operatorname{arcsec})\end{array}$ \\
\hline s0c0 & POINT & $05: 34: 32.65$ & 21.57 .16 .2 & 0.141 & {$[-0.018,0.066,1.504,-0.762]$} & 55369567 & - & - \\
s1c1 & GAUSSIAN & $05: 34: 23.88$ & 22.03 .22 .1 & 1.473 & {$[-0.945,1.228,1.427,-12.222]$} & 55369567 & 70.644 & 70.644 \\
\hline
\end{tabular}

Notes. The "Orientation" column (not shown) is always set at $0^{\circ}$.

(Dutch array). We release these high-fidelity, high-resolution models of these sources in the frequency range $30-77 \mathrm{MHz}$. A detailed analysis of each source is beyond the scope of this paper, and will be carried out in separate individual publications for each object. Nonetheless, in this section, we report an overview of our findings.

\subsection{Cassiopeia $A$}

LOFAR LBA data for Cassiopeia A, a $~ 330$ yr old supernova remnant, has been analysed recently by Arias et al. (2018). However, we note that the data and reduction methods presented in this work are new. The most striking feature of Cassiopeia A at low radio frequencies is the effect of internal free-free absorption from cold ( $100 \mathrm{~K}$; Arias et al. 2018; Oonk et al. 2017), unshocked supernova ejecta material. As a result, the central region of Cassiopeia A (interior to the bright shell) is less bright than in the gigahertz band. The presence of such internal absorption was first noted by Kassim et al. (1995), and further investigated by Delaney et al. (2014) with images down to $74 \mathrm{MHz}$.

The overall flux density within a beam centred on Cassiopeia A is always affected by free-free absorption by relatively cool free electrons between us and the source, as well as internal absorption in the central region. As a consequence, for a given beam, the flux density can be described as (Arias et al. 2018)

$S_{v}=S_{0}\left(\frac{v}{v_{0}}\right)^{-\alpha}\left[f+(1-f) \mathrm{e}^{-\tau_{v, \text { int }}}\right] \mathrm{e}^{-\tau_{v, \text { ISM }}}$,

where $f$, the flux fraction, comes from the unobscured part of the shell, and $(1-f)$ the covering fraction (i.e. the back side of the supernova-remnant shell); $\tau_{v \text {,int }}$ is the optical depth due to free-free absorption from the unshocked ejecta, and $\tau_{\nu, \text { ISM }}$ is the free-free absorption due to the free electrons between us and Cassiopeia A. The free-free absorption scales as $\tau_{v} \propto$ $v^{-2} T^{-3 / 2} n_{\mathrm{e}} \sum_{i} n_{i}$, which shows that the internal mass estimate is dependent on the temperature of the free electrons and the composition and degree of ionisation of the unshocked supernova ejecta. Moreover, clumping of the ejecta may seriously affect the relation between the internal, unshocked mass, and the internal free-free absorption.

The effect of the internal absorption is that the central part of Cassiopeia A is less bright below $100 \mathrm{MHz}$ than at high frequencies. Once $\tau_{v \text {,int }} \gg 1$ the total flux density continues to fall again as $S_{v} \propto v^{\alpha}$, but with a flux reduced by $(1-f)$ compared to the extrapolation from high frequencies, except that the external free-free absorption causes an overall reduction of the flux density. As a result, the maximum flux density of Cassiopeia A occurs around $20 \mathrm{MHz}$ (Baars et al. 1977).

The multi-channel LOFAR LBA data provide a more precise localisation of the effect and infer an unshocked ejecta mass of $(3 \pm 0.5) M_{\odot}$. We plan to update this result using the new calibration and data reduction procedures presented in this work (Arias et al., in prep.).

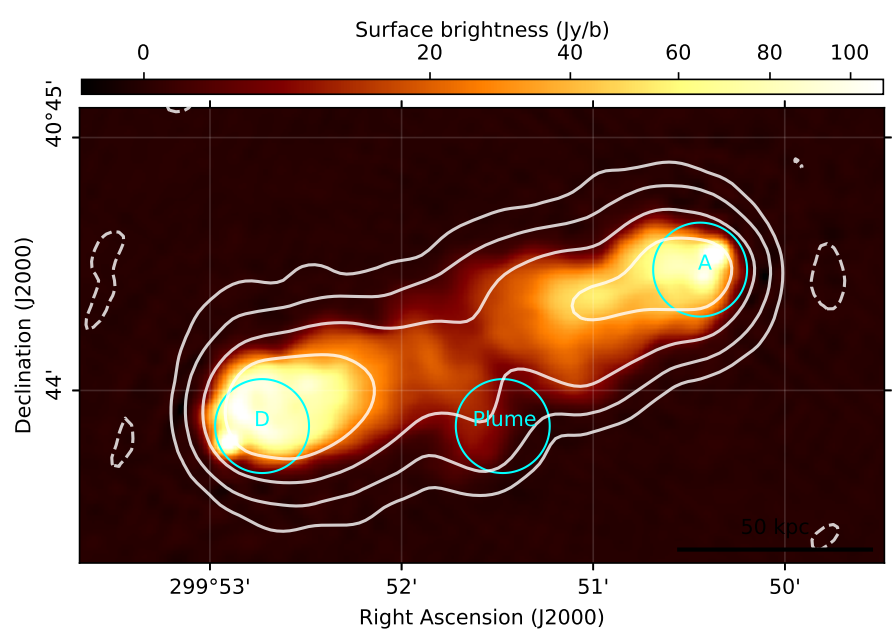

Fig. 2. LOFAR HBA image of Cygnus A (central frequency of $146 \mathrm{MHz}$ ). The resulting rms map noise is $43 \mathrm{mJybeam}^{-1}$ and the FWHM beam size is $3 " .8 \times 2$ ".7 (from McKean et al. 2016). Contours from the LBA map at $-0.3,10,30,100,300 \mathrm{Jy}^{\text {beam }^{-1}}$ are superimposed. The circles represent the regions where we extracted the LBA in-band spectral index.

\subsection{Cygnus $A$}

This is the first work examining this source both at this frequency and resolution. We report the work of Lazio et al. (2006), which reached a similar resolution of our LOFAR images at $74 \mathrm{MHz}$. The most striking feature in the new LOFAR LBA image of Cygnus $\mathrm{A}$ is the absence of hotspots that are seen at higher frequencies McKean et al. (2016). After convolving all of the LBA images to the same resolution, we attempted the extraction of the in-band spectral index $\alpha$ (with $s_{v} \propto v^{\alpha}$ ) in three regions with a size that is equivalent to the convolved beam. We positioned two regions close to the east and west edges of the source, and they gave spectral index values of $\alpha=0.46 \pm 0.05$ and $\alpha=0.25 \pm 0.05$, respectively, for hotspots A and D (defined in McKean et al. 2016, see Fig. 2) between $30 \mathrm{MHz}$ and $77 \mathrm{MHz}$. The third region was positioned at the source centre, close to the southern plume, which gave a spectral index of $\alpha=-0.82 \pm 0.05$ between 30 and $77 \mathrm{MHz}$. We note that beam dilution almost certainly biases the results towards steeper values in the case of the hotspots. To calculate the uncertainties, a conservative flux error of $10 \%$ in each measurement was added to the error estimated from the map noise.

As discussed by McKean et al. (2016), the spectral energy distribution in the hotspot regions $\mathrm{A}$ and $\mathrm{D}$ peaks between 140 and $160 \mathrm{MHz}$, and then starts decreasing towards lower frequencies. The two main models proposed to explain the turnover are as follows: (i) free-free absorption or synchrotron self-absorption processes within the hotspots or along the line of sight (Kassim 1989) and/or (ii) a cut-off in the electron energy distribution at low energies (Carilli et al. 1991). From 
the LOFAR HBA data, in combination with higher frequency data from the VLA, McKean et al. (2016) found that the strong turnover in the spectral index ruled out a cut-off in the electron energy distribution at low energies, and the limit in the spectral index provided by the new LOFAR LBA imaging is consistent with that conclusion. McKean et al. (2016) also found that the synchrotron self-absorption model was also unlikely, given the very large magnetic field strengths needed to cause such a turnover $(B \approx 1-2 \mathrm{G})$ relative to the modest magnetic field strength that is required by the synchrotron cooling model for both hotspots $(B \approx 150 \mu \mathrm{G})$. The free-free model was also challenging to explain the data since the implied electron densities $\left(n_{\mathrm{e}} \approx 2 \mathrm{~cm}^{-3}\right)$ should result in a significant de-polarisation of the emission seen at $\mathrm{GHz}$ frequencies, which is not the case. Only low-frequency observations of the hotspots in the LOFAR LBA can distinguish between different models. However, our resolution is not sufficient to constrain such models, and therefore observations with the international baselines, to achieve the arcsecond resolution needed, are planned. Nevertheless, our inverted spectrum for the hotspot regions confirms that some form of absorption must be at least partially responsible for the observed turnover.

The plume extending from the central part of the source towards the south is also visible and the in-band spectral index is in line with what is measured at higher frequencies by McKean et al. (2016). Finally, we report the detection of diffuse emission, with an extension $\sim 4^{\prime}$ towards the north-east of Cygnus A. The classification of this source is difficult because of the dynamic range of the image. It could be a background radio galaxy or some emission related to the intra-cluster medium dynamics.

\subsection{Taurus $A$}

This radio source is associated with the Crab Nebula (see Hester 2008; Bühler \& Blandford 2014, for a review), which is the supernova remnant of SN 1054 (e.g. Stephenson \& Green 2002). However, most of the electromagnetic radiation is coming from the pulsar wind nebula (PWN) that is powered by the Crab Pulsar (PSR B0531+21), which has a period of $33 \mathrm{~ms}$, and a rotational energy loss rate of $\dot{E}=4.6 \times 10^{38} \mathrm{erg} \mathrm{s}^{-1}$. Taurus A is unique in that synchrotron emission is dominating the spectrum from low radio frequencies up to $\sim 100 \mathrm{MeV}\left(\sim 10^{22} \mathrm{~Hz}\right)$. Synchrotron emission even dominates the optical and UV band (Miller 1978), but the optical also reveals strong line emission from the filaments of ionised supernova ejecta. The radio synchrotron spectrum has a spectral index of $\alpha \approx-0.3$ (Green 2019), but in the optical the spectral index is closer to $\alpha \approx-0.8$ (Miller 1978) and it is even steeper in X-rays with $\alpha \approx-1.1$ (Madsen et al. 2017). The spectral break between the radio and optical can be understood as due to synchrotron cooling, giving a magnetic field of $B \approx 100-200 \mu \mathrm{G}$ and an age of $\sim 950 \mathrm{yr}$, but the steep X-ray spectrum is not well understood. One suggestion is that there are two populations of relativistic electron/positron: one responsible for the radio emission and another for the UV/X-ray (e.g. emission Meyer et al. 2010). The radio population could be the result of a past injection of particles; (i.e. "relic electrons/positrons" Atoyan \& Aharonian 1996), or two different electron/positron acceleration mechanisms, such as reconnection for the low-energy population associated with the radio emission, and diffusive shock acceleration for the higher energy particles responsible for X-rays. To complicate things, the injection of fresh electron/positrons seems to occur on the inside of the bright optical/X-ray torus (Hester 2008, and reference therein), but some X-ray emission is also associated with two jets that are roughly orientated south-east to north-west.

The radio emission from Taurus $A$ in the LOFAR LBA, as seen in Fig. 1, is elongated, in the south-east to northwest direction. This is similar to higher frequency maps (e.g. Bietenholz \& Nugent 2015, for a 5.5 GHz VLA map). However, what is at least qualitatively different between the low- and highfrequency radio maps is that at low frequencies there seems to be relatively less emission from the torus region and more emission associated with the "jets", suggesting that these two components have different spectral indices, which could potentially shed new light into whether the PWN consists of a single electron/positron population with a complicated energy distribution or two or even more populations with different physical origins. We caution, however, that this needs to be further investigated as the dynamical range and the uv-coverage of the LOFAR LBA and $5.5 \mathrm{GHz}$ VLA maps are not similar, requiring care to assess quantitative differences. We will come back to this issue in a future paper dedicated to the LOFAR LBA observation of Taurus A presented in this work.

Finally, we note that the centre of Taurus A is dominated by the emission from the steep spectrum of pulsar with an inband spectral index of $\alpha=-1.50 \pm 0.05$, in line with previous measurements (Bridle 1970).

\subsection{Virgo $A$}

This is the most extended of the A-team sources, reaching an apparent scale of about $15^{\prime}$. Virgo A is the radio emission associated with the active galaxy M 87 and is famous for hosting one of the best-studied supermassive black holes (recently imaged by Event Horizon Telescope Collaboration 2019). The central cocoon, which at these frequencies accounts for just $\sim 30 \%$ of the total source flux density, hosts the well-known one-sided jet and morphologically resembles an FR II radio galaxy. However, Virgo A emission extends well beyond the central cocoon and the majority of the flux density comes from a relatively low-surface brightness envelope filled with filamentary structures. In this region, clear connection between the radio and the $\mathrm{X}$-ray emission shows one of the best examples of active galactic nucleii feedback in action, where cold gas is uplifted by buoyantly rising bubbles towards the outskirts of the galaxy potential well (Forman et al. 2007). The external boundaries of the source appear well confined even at ultra-low frequencies; this was already observed at higher frequencies (Owen et al. 2000; de Gasperin et al. 2012). The resolution of these new maps will enable the first detailed spectral study of the source envelope and of the embedded filamentary structures. This analysis will be part of a future publication.

Acknowledgements. The Leiden LOFAR team gratefully acknowledge support from the European Research Council under the European Unions Seventh Framework Programme (FP/2007-2013)/ERC Advanced Grant NEWCLUSTERS-321271. AB acknowledges financial support from the Italian Minister for Research and Education (MIUR), project FARE SMS, code R16RMPN87T and from the ERC-Stg DRANOEL, no. 714245. LOFAR, the Low Frequency Array designed and constructed by ASTRON, has facilities in several countries, that are owned by various parties (each with their own funding sources), and that are collectively operated by the International LOFAR Telescope (ILT) foundation under a joint scientific policy. This research has made use of NASA's Astrophysics Data System. 


\section{References}

Arias, M., Vink, J., de Gasperin, F., et al. 2018, A\&A, 612, A110 Atoyan, A. M., \& Aharonian, F. A. 1996, MNRAS, 278, 525

Baars, J. W. M., Genzel, R., Pauliny-Toth, I. I. K., \& Witzel, A. 1977, A\&A, 61, 99

Bailer-Jones, C. A. L., Rybizki, J., Fouesneau, M., Mantelet, G., \& Andrae, R. 2018, ApJ, 156, 58

Bietenholz, M. F., \& Nugent, R. L. 2015, MNRAS, 454, 2416

Bowman, J. D., Rogers, A. E., Monsalve, R. A., Mozdzen, T. J., \& Mahesh, N. 2018, Nature, 555, 67

Bridle, A. H. 1970, Nature, 225, 1035

Brienza, M., Godfrey, L., Morganti, R., et al. 2016, A\&A, 585, A29

Briggs, D. S. 1995, Bull. Am. Astron. Soc., 27, 112

Bühler, R., \& Blandford, R. 2014, Rep. Progr. Phys., 77, 6

Carilli, C. L., Perley, R. A., Dreher, J. W., \& Leahy, J. P. 1991, ApJ, 383, 554

de Gasperin, F., Orrú, E., Murgia, M., et al. 2012, A\&A, 547, A56

de Gasperin, F., Intema, H. T., Shimwell, T. W., et al. 2017, Sci. Adv., 3, e1701634

de Gasperin, F., Dijkema, T. J., Drabent, A., et al. 2019, A\&A, 622, A5

Delaney, T., Kassim, N. E., Rudnick, L., \& Perley, R. A. 2014, ApJ, 785, 7

Event Horizon Telescope Collaboration (Akiyama, K., et al.) 2019, ApJ, 875, L1

Forman, W., Jones, C., Churazov, E., et al. 2007, ApJ, 665, 1057

Green, D. A. 2019, JApA, 40, 36

Hamaker, J. P., Bregman, J. D., \& Sault, R. J. 1996, A\&AS, 117, 137

Hester, J. J. 2008, ARA\&A, 46, 127

Hoang, D. N., Shimwell, T. W., Stroe, A., et al. 2017, MNRAS, 471, 1107

Hurley-Walker, N., Callingham, J. R., Hancock, P. J., et al. 2017, MNRAS, 464, 1146

Intema, H. T., Jagannathan, P., Mooley, K. P., \& Frail, D. A. 2017, A\&A, 598, A78

Kassim, N. E. 1989, ApJ, 347, 915

Kassim, N. E., Perley, R. A., Dwarakanath, K. S., \& Erickson, W. C. 1995, ApJ, 455, L59

Kazemi, S., Yatawatta, S., Zaroubi, S., et al. 2011, MNRAS, 414, 1656

Lazio, T. J. W., Cohen, A. S., Kassim, N. E., et al. 2006, ApJ, 642, L33

Madsen, K. K., Forster, K., Grefenstette, B. W., Harrison, F. A., \& Stern, D. 2017, ApJ, 841, 56

Markevitch, M., Sarazin, C. L., \& Vikhlinin, A. 2002, ApJ, 521, 526

McKean, J. P., Godfrey, L. E. H., Vegetti, S., et al. 2016, MNRAS, 463, 3143

Meyer, M., Horns, D., \& Zechlin, H. S. 2010, A\&A, 523, A2

Miller, J. S. 1978, ApJ, 220, 490

Noordam, J. E. 2004, Proc. SPIE, 5489, 817

Offringa, A. R., de Bruyn, A. G., Zaroubi, S., \& Biehl, M. 2010, ArXiv e-prints [arXiv:1007.2089]

Offringa, A. R., McKinley, B., Hurley-Walker, N., et al. 2014, MNRAS, 444, 606

Oonk, J. B., van Weeren, R. J., Salas, P., et al. 2017, MNRAS, 465, 1066

Owen, F., Eilek, J., \& Kassim, N. 2000, ApJ, 543, 611

Patil, A. H., Yatawatta, S., Koopmans, L. V. E., et al. 2017, ApJ, 838, 65

Perley, R. A., \& Butler, B. J. 2017, ApJS, 230, 7

Reed, J. E., Hester, J. J., Fabian, A. C., \& Winkler, P. F. 1995, ApJ, 440, 706

Saxena, A., Marinello, M., Overzier, R. A., et al. 2018, MNRAS, 480, 2733

Shimwell, T. W., Röttgering, H. J. A., Best, P. N., et al. 2017, A\&A, 598, A104

Smirnov, O. M. 2011, A\&A, 527, A106

Smirnov, O., \& Tasse, C. 2015, MNRAS, 449, 2668

Stephenson, F. R., \& Green, D. A. 2002, Historical Supernovae and their Remnants (Oxford: Oxford University Press), 252

Van der Tol, S. 2009, PhD Thesis, Delft University of Technology, The Netherlands

van Haarlem, M. P., Wise, M. W., Gunst, A. W., et al. 2013, A\&A, 556, A2

Vinyaikin, E. N. 2014, Astron. Rep., 58, 626

1 Hamburger Sternwarte, Universität Hamburg, Gojenbergsweg 112, 21029 Hamburg, Germany e-mail: fdg@hs.uni-hamburg.de

2 Anton Pannekoek Institute for Astronomy, University of Amsterdam, Science Park 904, 1098 XH Amsterdam, The Netherlands

3 GRAPPA, University of Amsterdam, Science Park 904, 1098 XH Amsterdam, The Netherlands

4 SRON, Netherlands Institute for Space Research, Utrecht, The Netherlands
Kapteyn Astronomical Institute, University of Groningen, PO Box 800, 9700 AV Groningen, The Netherlands

6 ASTRON - the Netherlands Institute for Radio Astronomy, Oude Hoogeveensedijk 4, 7991 PD Dwingeloo, The Netherlands

7 Eindhoven University of Technology, PO Box 513, 5600 MB Eindhoven, The Netherlands

8 Leiden Observatory, Leiden University, Niels Bohrweg 2, 2333 CA Leiden, The Netherlands

9 Department of Physics \& Electronics, Rhodes University, 6139 Grahamstown, South Africa

10 South African Radio Astronomy Observatory, 7925 Observatory, Cape Town, South Africa

11 Thüringer Landessternwarte, Sternwarte 5, 07778 Tautenburg, Germany

12 Astronomical Observatory, Jagiellonian University, ul. Orla 171, 30-244 Kraków, Poland

13 LPC2E - Université d'Orléans/CNRS, Orléans, France

14 Dipartimento di Fisica e Astronomia, Universitá di Bologna, Via P. Gobetti 93/2, 40129 Bologna, Italy

15 INAF - Istituto di Radioastronomia, Bologna Via Gobetti 101, 40129 Bologna, Italy

16 LESIA \& USN, Observatoire de Paris, CNRS, PSL, SU/UP/UO, 92195 Meudon, France

17 Department of Space, Earth and Environment, Chalmers University of Technology, Onsala Space Observatory, 43992 Onsala, Sweden

18 Jodrell Bank Centre for Astrophysics, Department of Physics \& Astronomy, The University of Manchester, Alan Turing Building, Oxford Road, Manchester M13 9PL, UK

19 Department of Physics, The George Washington University, 725 21 st Street NW, Washington, DC 20052, USA

20 Astronomy, Physics, and Statistics Institute of Sciences (APSIS), The George Washington University, Washington, DC 20052, USA

21 Erlangen Center for Astroparticle Physics (ECAP), FriedrichAlexander-Universität Erlangen-Nürnberg, 91058 Erlangen, Germany

22 DESY, Platanenallee 6, 15738 Zeuthen, Germany

23 Space Radio-Diagnostics Research Centre, University of Warmia and Mazury in Olsztyn, Prawochenskiego 9, 10-720 Olsztyn, Poland

24 Leibniz-Institut für Astrophysik Potsdam (AIP), An der Sternwarte 16, 14482 Potsdam, Germany

25 CSIRO Astronomy and Space Science, PO Box 1130, Bentley, WA 6102, Australia

26 Research School of Astronomy and Astrophysics, Mount Stromlo Observatory, Cotter Road, Weston Creek ACT 2611, Australia

27 Shell Technology Center, Bangalore 562149, India

28 Max Planck Institute for Astrophysics, Karl-Schwarzschild-Str. 1, 85748 Garching, Germany

29 Fakultät für Physik, Universität Bielefeld, Postfach 100131, 33501 Bielefeld, Germany

30 USN, Station de Radioastronomie de Nançay Observatoire de Paris route de Souesmes, 18330 Nançay, France

31 Univ. Lyon, Univ. Lyon1, Ens de Lyon, CNRS, Centre de Recherche Astrophysique de Lyon UMR5574, 9 Av Charles André, 69230 Saint-Genis-Laval, France

32 Department of Astrophysics/IMAPP, Radboud Universiteit, PO Box 9010, 6500 GL Nijmegen, The Netherlands

33 Nikhef, Science Park 105, 1098 XG Amsterdam, The Netherlands

34 Vrije Universiteit Brussel, Physics Department, Pleinlaan 2, 1050 Brussels, Belgium

35 Max-Planck-Institut für Radioastronomie, Auf dem Hügel 69, 53121 Bonn, Germany

36 IBCH PAS Poznan Supercomputing and Networking Center (PSNC), Poznan, Poland

37 International Centre for Radio Astronomy Research, Curtin University, GPO Box U1987, Perth, WA 6845, Australia 